\title{
Efficient video watermarking with SWT and empirical PCA based decoding
}

\author{
Teena M. Thomas
}

\begin{abstract}
Digital content piracy is one of the major crimes in the present world. Protection of digital content like music, video and images has become a major problem. Watermarking is one of the methods to protect digital content against unauthorized copying. In watermarking procedure, generally, watermark is embedded before it is going to be distributed over different communication channels. This paper presents a novel technique for embedding a imageldata into video frames. Video Watermarking is a process by which user specified data is hidden or embedded into another signal (Video Signal) by the watermark embedding process. Afterwards the recovery of the watermark is achieved with the help of the watermark extraction process. The proposed scheme is an imperceptible and a robust hybrid video watermarking scheme. PCA is applied to each block of the any bands which result from Stationary Wavelet transform of every video frame. The watermark is embedded into the first principal components of the any blocks in different ways. Combining the two transforms improved the performance of the watermark algorithm. The scheme is tested by applying various attacks. Experimental results show no visible difference between the watermarked frames and the original frames. In our paper, to embed the watermark, stationary wavelet transform (SWT) is used in combination with principal component analysis (PCA) in order to increase the robustness against many attacks, to increase the amount of payload and also the processing speed.
\end{abstract}

Index Terms: Video Watermarking, Stationary Wavelet Transform, Principal Component Analysis.

\section{Introduction}

The rapid growth of network distributions of images and video, there is a need for copyright protection against piracy. For this purpose we are using video watermarking (VM). Different digital watermarking schemes have been proposed to address this issue of ownership identification. Digital watermarking is a process by which user specified signal(Watermark) is hidden or embedded into another signal(Video Signal) by the watermark embedding process. Afterwards the recovery of the watermark is achieved with the help of the watermark extraction processit has various applications, such as steganography, fingerprinting, copyright protection, and so on. Various aspects of watermarking systems include transparency robustness, capacity, security, and computational complexity of the embedding and decoding process. Considering video as a sequence of images, one could simply put data in each frame independently using still image watermarking algorithms. This is one of the most straightforward ways for data embedding in video.

Two major issues in hiding information are you want to protect the data from malicious use because you do not want any one to even know about its existence to avoid observation by unintended recipients "Security through obscurity" Information hiding can be mainly divided into three processes - cryptography, stenography and watermarks. Cryptography is the process of converting information to an unintelligible form so that only the authorized person with the key can decipher it. As many advances were made in the field of communication it became rather simple to decrypt a cipher text. Hence more sophisticated methods were designed to offer better security than what cryptography could offer. This led to the discovery of stenography and watermarking. Stenography is the process of hiding information over a cover object such that the hidden information cannot be perceived by the user. Thus even the existence of secret information is not known to the attacker. Watermarking is closely related to stenography, but in watermarking the hidden information is usually related to the cover object. Hence it is mainly used for copyright protection and owner authentication.

Every day tons of data are embedded in digital media or distributed over the internet. The data so distributed can easily be replicated without error, putting the rights of their owners at risk. Even when encrypted for distribution, Data can easily be decrypted and copied. One way to discourage illegal duplication is to insert information known as watermark, in potentially vulnerable data in such a way that it is impossible to separate the watermark from the data. These challenges motivated researchers to carry out intense research in the field of watermarking. A watermark is a form, image or text that is impressed onto paper, which provides evidence of its authenticity. Digital watermarking is an extension of the same concept. There are two types of watermarks: visible watermark and invisible watermark. In this project we have concentrated on implementing watermark on image. The main consideration for any watermarking scheme is its robustness to various attacks. Watermarking dependency on the original image increases its robustness but at the same time we need to make sure that the watermark is imperceptible. In this project an invisible watermarking technique (least significant bit) is 
implemented. An attack is also implemented in the visible watermarked image by adding a random noise to the watermarked image. The watermarked image is then compressed and decompressed using JPEG compression. Finally noise is removed and the images are separated from the recovered watermarked image.

Depending upon the information needed for watermark decoding they are clsassified as blind,semi blind and non blind .blind system do not acess the orginal host data and the semi blind system can be imagined as a communication system with side information non-blind systems need to have the original host data at the decoder in order to decode the watermark.data can be transparently embedded in to the video and extract the data in a robust manner after many alteration that is known as attacks.for an application in which a peron create a 5 minute video clip with intend to upload it to the youtube for advertising as he encrypted the watermarked data and store the side information in a secure location and registred with the legal authority with semi blind method so no one can acess the rightful ownership if he get an illegal copy .

\section{Related Work}

In this section we briefly describe the three related topics:1)A Block Based Novel Digital Video Watermarking Scheme Using Dct 2) A New Scheme For Robust Blind Digital Video Watermarking 3)Efficient Video Watermarking With Pca-Based Decoding

\section{A ) A Block Based Novel Digital Video Watermarking Scheme Using Dct}

Different digital watermarking schemes have been proposed to address this issue of ownership identification. The video watermark is robust against the attack of frame dropping, averaging and statistical analysis. It leads to broad curiosity in multimedia security and multimedia copyright protection. The most important issue in $\mathrm{VM}$ are the invisibility of the watermark and the resilience of watermarking to attacks. Watermarking techniques are classified into three categories. They are Spatial Domain Method(SDM), Transform Domain Method(TDM) and Compressed Domain Method(CDM). Here explained about to transform domain method. This method used the discrete cosine transform(DCT), Discrete Fourier Transform(DFT) and Discrete Wavelet Transform(DWT) for watermark embedding.DWT are more popularity due to their spatial localization, frequency spread etc.[1]. But DCT watermarking was to human perception model to modify low coefficients of DCT blocks.It has a larger embedding capacity and robustness. This technique provides better results with high accuracy. Discrete cosine transform is a process which converts a sequence of data points in the spatial domain to a sum of sine and cosine waveforms with different amplitudes in the frequency domain.

The DCT is a linear transform, which maps a n-dimensional vector to set of $n$ coefficients. A linear combination of $\mathrm{n}$ known basis vectors weighted with the $\mathrm{n}$ coefficients will result in the original vector. The known basis vectors of transforming from this class are "sinusoidal", which means that they can be represented by sinus shaped waves or, in other words, they are strongly localized in the frequency spectrum. Therefore one speaks about the transformation to the frequency domain. The most popular member of this class is the Discrete Fourier Transform (DFT). The difference between DCT and DFT is that DFT applies to complex numbers, while DCT uses just real numbers. For real input data with even symmetry DCT and DFT are equivalent. There are eight different variants of DCT. There is a very slight modification between these eight variants. In JPEG compression the input data are two-dimensional, presented in $8 \times 8$ blocks. There's a need of using twodimensional DCT. Since each dimension can be handled separately, the two-dimensional DCT follows straightforward form the one-dimensional DCT. A one-dimensional DCT is performed along the rows and then along the columns, or vice versa. The formula used for one-dimensional DCT: Wavelet Transform based Watermarking The Fourier transform is ananalys is of global frequency content in the signal. There are applications in digital image processing wherein we need the localized frequency components. This can be done by using the Short Time Fourier Transform. This is similar to the concept of using windowing functions. This equation transforms the signal $\mathrm{f}(\mathrm{x})$ in a small window around. The STFT is then performed on the signal and local information is extracted. The wavelet transform based watermarking technique divides the image into four sidebands with a low resolution approximation of the tile component and the component's 'horizontal, vertical and diagonal frequency characteristics. The process can then be repeated iteratively to produce $\mathrm{N}$ scale transform. One of the many advantages over the wavelet transform is that it is believed to more accurately model aspects of the human visual system (HVS) as compared to the DCT. This allows us to use higher energy watermarks in regions that the HVS is known to be less sensitive to,such as higher resolution detail bands $\{\mathrm{LH}, \mathrm{HL}, \mathrm{HH})$. Embedding watermarks in these regions allow us to increase the robustness of our watermark, at little to no additional impact on image quality. One of the most straightforward techniques is to use an embedding technique similar to that used in the DCT. In the Wavelet Domain, where Wi watermark to be embedded, and $\alpha$ scaling factor.To detect the watermark the same process as that used in DCT is implemented.Furthermore, as the embedding uses the values of the transformed value in embedded, the embedding process would be rather adaptive; storing the majority of the watermark in the larger coefficients. 


\section{c) A New Scheme For Robust Blind Digital Video Watermarking}

Watermarking is a concept of embedding a special pattern, watermark, into a document Watermarking is a key process for the protection of copyright ownership of electronic data.

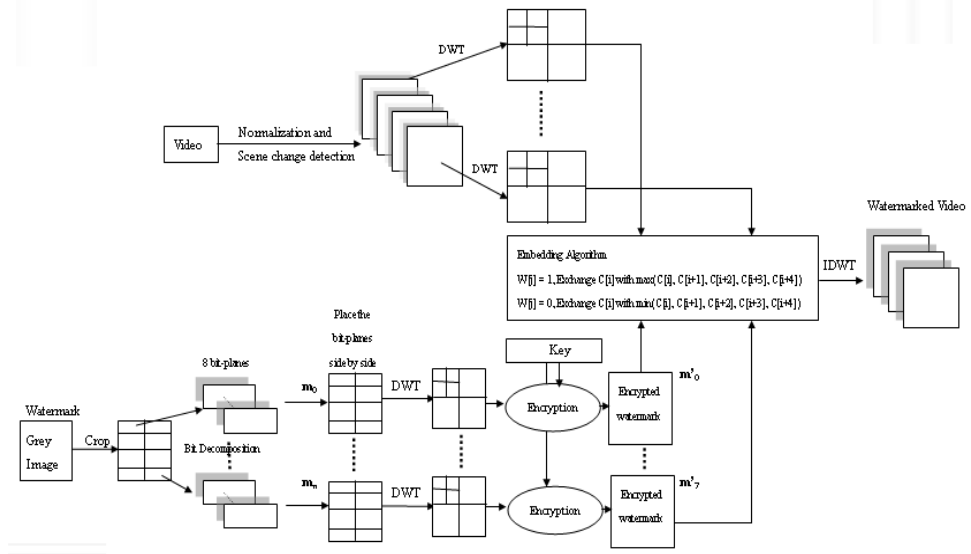

Fig.1.Overview of the watermarking process

\section{a) Video Preprocess DWT \& Scene Change Detection}

Video frames are normalized to 256 X 256 and transformed to wavelet domain Each scene is embedded with a same watermark, so it can prevent attackers from removing the watermark by frame dropping Independent watermark used for successive different scene can prevent attackers from colluding with frames from completely different scenes

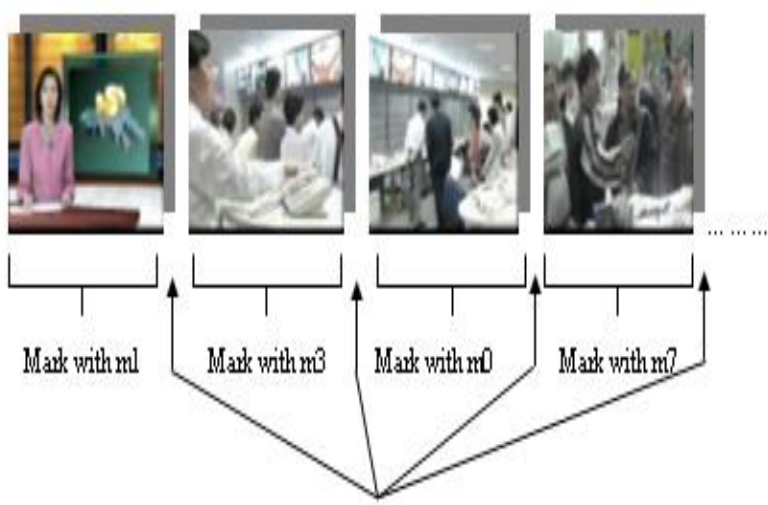

Scene change occur

b)Watermark Preprocess

Scale the watermark to a particular size with the following equations

- $\mathbf{2}^{\mathrm{n}} \leqq \mathrm{m}, \mathbf{n}>\mathbf{0}$

- $\mathbf{p}+\mathbf{q}=\mathbf{n}, \mathbf{p}$ and $\mathbf{q}>\mathbf{0}$

- Size of image $=64 \square 2^{p} \times 64 \square 2^{q}$

Divide the image into $2^{\mathrm{n}}$ small images with size 64 X 64 principle of comparing the color content of candidate regions to a reference color histogram, we embed it within a sequential Monte Carlo framework.

$\mathrm{M}=10, \mathrm{n}=3, \mathrm{p}=1, \mathrm{q}=2$

Different frames using different watermarks can make the watermarks resistant to attacks by frame averaging Hard to reconstruct the watermark without knowledge This requires the building of a color likelihood based on color histogram distances.

\section{c)Watermark Detection}

if $\mathrm{WC}[\mathrm{i}]>\operatorname{median}(\mathrm{WC}[\mathrm{i}]$,

$\mathrm{WC}[\mathrm{i}+1], \mathrm{WC}[\mathrm{i}+2]$,

$\mathrm{WC}[\mathrm{i}+3], \mathrm{WC}[\mathrm{i}+4]), \mathrm{W}[\mathrm{j}]=1$

else

$\mathrm{W}[\mathrm{j}]=0$ 


\section{D) video watermarking with empirical pca-based decoding}

This project presented an efficient video watermarking technique using discrete wavelet transform (DWT) to protect the copyright protection of digital images. The efficiency of the video watermarking technique is achieved with the aid of the following two major steps.

1) Watermark Embedding process

2) Watermark Extraction process

Before embedding watermark pixels into the input video sequences, the following process should carry out to enhance the security of the hiding information as well as to improve the efficiency of our proposed approach. The fundamental task of performing the video processing application like video watermarking and video retrieval is video shot segmentation. The original input video sequence is first segmented into nonoverlapping units, called shots/cubes that depict different actions. Each shot is characterized by no significant changes in its content which is determined by the background and the objects present in the scene. Here, we have used Discrete wavelet Transform and correlation measure to identify the number of frames involved in each shot. At first, the first and second frame is divided into a set of blocks of sizes and DwT is iapplied to every block of the frame.then we have to take the first principal component analysis for the auxillary bit calculation to identify where we embedded the data If we embedded the data then aux bit shows +1 Otherwise it shows -1 .for data extraction we perform inverse 2 -d wavelet transform and reconstruct the orginal image.

\section{Proposed Method}

We know that the DWT suffers a drawback: the DWT is not a time-invariant transform. This means that, even with periodic signal extension, the DWT of a translated version of a signal X is not, in general, the translated version of the DWT of X. The Stationary wavelet transform is a wavelet transform algorithm designed to overcome the lack of translation-invariance of the discrete wavelet transform (DWT). Translationinvariance is achieved by removing the downsamplers and upsamplers in the DWT. The SWT is an inherently redundant scheme as the output of each level of SWT contains the same number of samples as the input so for a decomposition of $\mathrm{N}$ levels there is a redundancy of $\mathrm{N}$ in the wavelet coefficients. Many DCT or DWT based algorithms can be used to embed the watermark in the host image. The experimental results with the combination of SWT and bit orthogonal wavelet provide more robustness against many of the attacks with better PSNR value when compared with the same methods using DWT and DCT Therefore SWT is preferred over DWT in this paper. The PSNR is improved since stationary wavelet transform (SWT) is designed to overcome the lack of translation invariance of the discrete wavelet transform (DWT) Stationary Wavelet Transform performs a multilevel 1-D stationary wavelet decomposition using either a specific orthogonal wavelet or specific orthogonal wavelet decomposition filters. $\mathrm{N}$ must be a strictly positive integer and length $(\mathrm{X})$ must be a multiple of $2 \mathrm{~N}$.

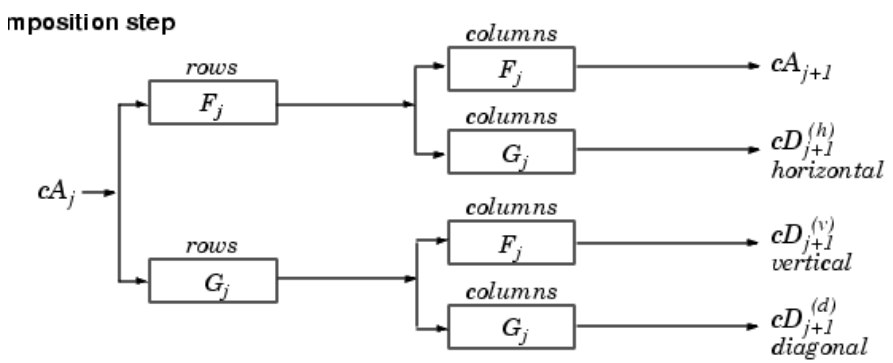

Fig 3.Block diagram of 2 level swt decomposition

In this algorithm, using the stationary wavelet transform, the image is decomposed into high and low frequency components, called first level decomposition. Once again the low frequency components of first level are decomposed into low and high frequency components, called second level decomposition. The preferred watermark is embedded into the second level decomposed low frequency components for robustness. As shown in fig-2, filters in each level are upsampled versions of the previous. In this paper we prefer SWT over the other wavelet transformation techniques because the SWT procedures do not include any down sampling steps and SWT employs null placing procedure instead of down sampling steps which is found in other wavelet transformation techniques.We simply apply appropriate high and low pass filters to the data at each level to produce two sequences. Instead of decimation, two new sequences will be generated of same length as the original sequence. Instead, filters are modified at each level, by padding them with zeros. 


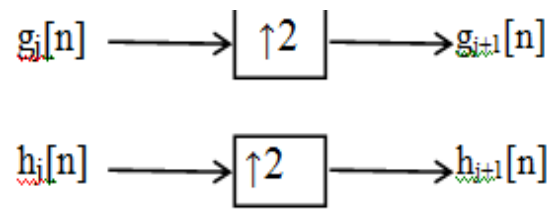

Fig 4.Swt upsampling filters

\subsection{Watermark Embedding}

The present system deals with processing of watermark embedding of image/data into the video In this proposed method, the usage of SWT in combination with orthogonal filters helps to increase the PSNR compared to DWT. More over SWT also allows embedding large amount of payload (watermark) that is almost half the size of the host image. Another important advantage of the SWT is the watermark need not to be strictly square in dimensions which is a mandatory condition in many transformation techniques like DCT, DWT etc. Even a rectangular watermark can be able to hide inside the host.The processing speed is much more compared to DWT techniques, which is the important characteristic in real time applications. This is enabled with the help of SWT. Before embedding the watermark into the host image, the image or data has to be in encrypted form.The proposed watermarking scheme is based on combining two transformations; the DWT and the PCA. The block diagrams of embedding and extraction algorithms are shown in Fig. 5 and Fig. 6. In our method, video frames are taken as the input, and watermark is embedded in each frame by altering the wavelet coefficients of selected SWT subbands, followed by performing the PCA transformation on the selected subbands.

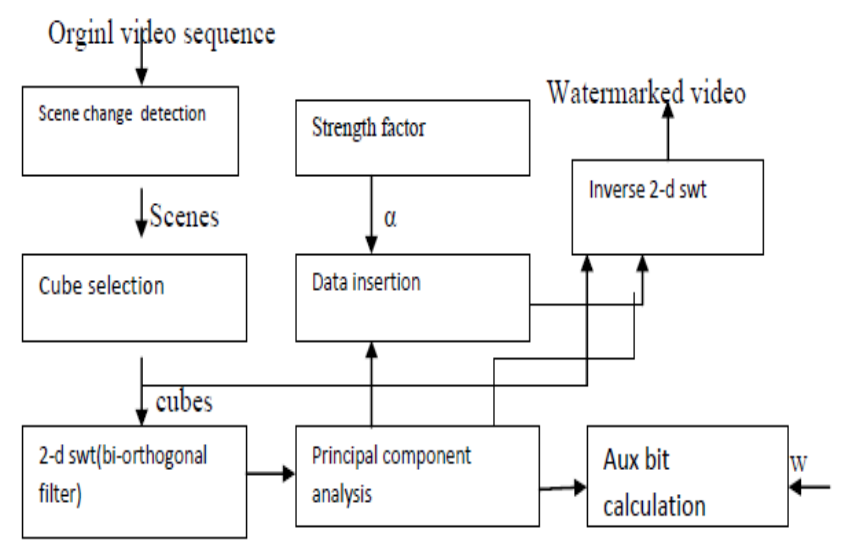

Fig. 5.Watermark embedding

Before embedding watermark pixels into the input video sequences, the following process should carry out to enhance the security of the hiding information as well as to improve the efficiency of our proposed approach. The process includes,

1) Shot segmentation of video sequences

2) Bit plane slicing of a grayscale image

3) Pixel permutation

4) Decomposition of an video frames using 2-level SWT

\section{1) Shot segmentation of video sequences}

The fundamental task of performing the video processing application like video watermarking and video retrieval is video shot segmentation. The original input video sequence is first segmented into nonoverlapping units, called shots/cubes that depict different actions. Each shot is characterized by no significant changes in its content which is determined by the background and the objects present in the scene. Here, we have used Stationary wavelet Transform and correlation measure to identify the number of frames involved in each shot. At first, the first and second frame is divided into a set of blocks of sizes and swt is applied to every block of the frame.then we have to take the first principal component analysis for the auxillary bit calculation to identify where we embedded the data. In the proposed algorithm, one bit for each cube is stored as side information: if the cube is watermarked, this bit is 1 ,otherwise it is 0 . Hence, the size of side information is equal to the number of cubes in the video.If we embedded the data then aux bit shows +1

Otherwise it shows -1 


$$
\begin{aligned}
& \mathrm{W}=+1 \text { Auxbit }=+1 \\
& \mathrm{~W}=-1 \text { Auxbit }=-1
\end{aligned}
$$

\section{2) Bit plane slicing of a grayscale image}

Bit-Plane Slicing is a technique in which the image is sliced at different planes. Instead of highlighting gray level images, highlighting the contribution made to the total image appearance by specific bits might be desired. Imagine the image is composed of 8 bits, 1-bit planes ranging from bit plane1-0 (LSB) to bit plane 7 (MSB). In terms of 8-bits bytes, plane 0 contains all lowest order bits in the bytes comprising the pixels in the image and plane 7 contains all high order bits [5]. Often by isolating particular bits of the pixel values in an A. The advantage of doing this method is to get the relative importance played by each bit of the image

\section{3) Pixel permutation}

After the bit plane slicing process, the sliced images are allowed to permute each pixel value to enhance the security of the hiding information. In this scheme, each group of pixels is taken from the image. The pixels in the group are permuted using the key selected from the set of keys. The size of the pixel group is same as the length of the keys, and all the keys are of the same length. If the length of the keys is more than the size of pixel group, the perceptual information reduces. In this, the group of pixels is taken along the row without the loss of generality, i.e., the column wise procedure would yield the same kind of results [6].

\section{4) Decomposition of an video frames using 2-level swt}

In the proposed approach, each video frame is divided into non-overlapping blocks and 2D SWT transform is applied to each block. A sequence of co-located blocks in a group of consecutive frames is referred to as a "cube." A set of cubes is then chosen, and data are embedded in LL coefficients of the blocks in the chosen cubes. The strength of data embedding is selected adaptively based on the energy of high frequency components and visual saliency. The decoding algorithm makes use of the data projected onto the first principal component.

\subsection{Video Extraction Process}

To extract the watermark sequence, watermarked cubes are distinguished from non-watermarked cubes via side information. The watermarked sequence is subject to possibleattack(s). After embedding the grayscale watermark image pixels into the original video sequence, we have extracted the embedded watermark image without affecting the original video.

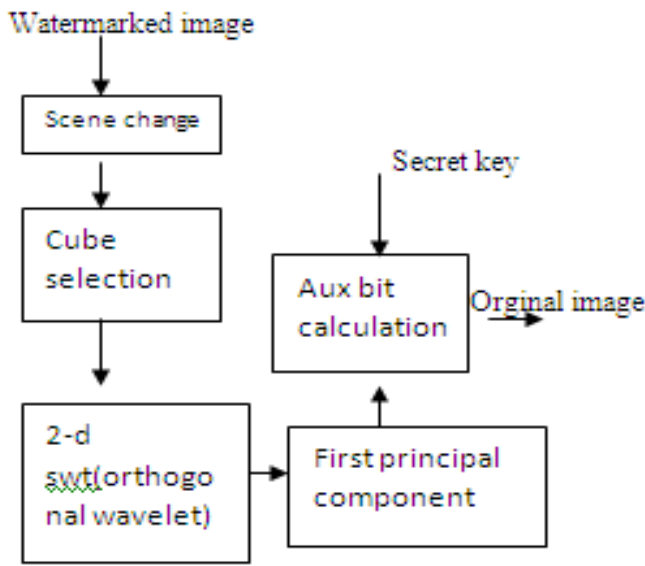

Fig .3.2 Watermark extraction

\section{Results}

The main criteria in the performance analysis are the time required for embedding the watermark/logo image for real time processing and to achieve high PSNR value. The time can further be reduced by using the appropriate digital image/signal processor. The PSNR is the objective criteria used to measure the quality of the watermarked image. Similarly the quality of the extracted watermark is measured by comparing it with the original watermark and is called similarity measure.To compute the PSNR, the mean-squared error is first measured. The performance has been evaluated in terms of the imperceptibility and robustness against various attacks. To measure the robustness, several experiments had been done. The watermarked frame was subjected to different attacks. The chosen attacks were MPEG4, JPEG, scaling, adding noise, filtering, rotation, histogram equalization, contrast adjustment, gamma correction, cropping, frame averaging and the frame dropping 

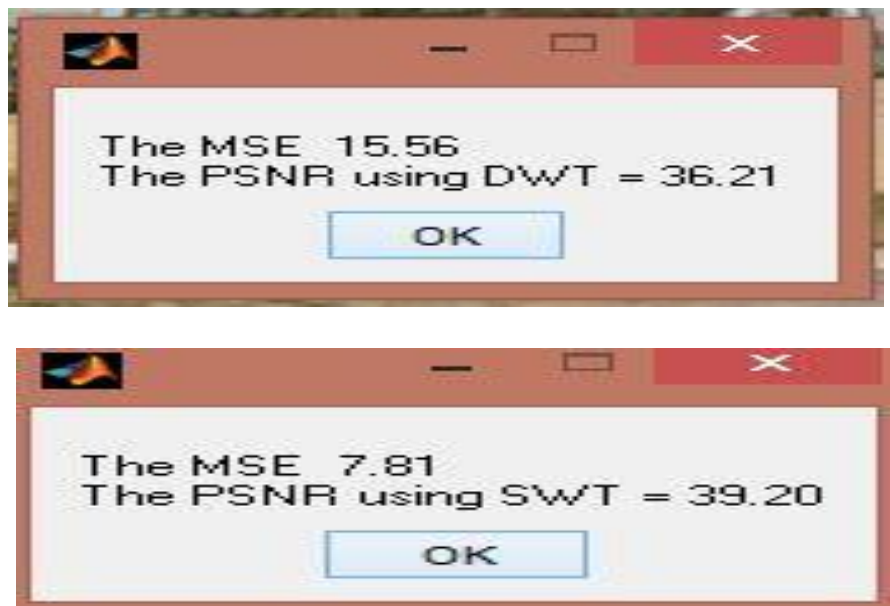

Figure 1.Performance comparison

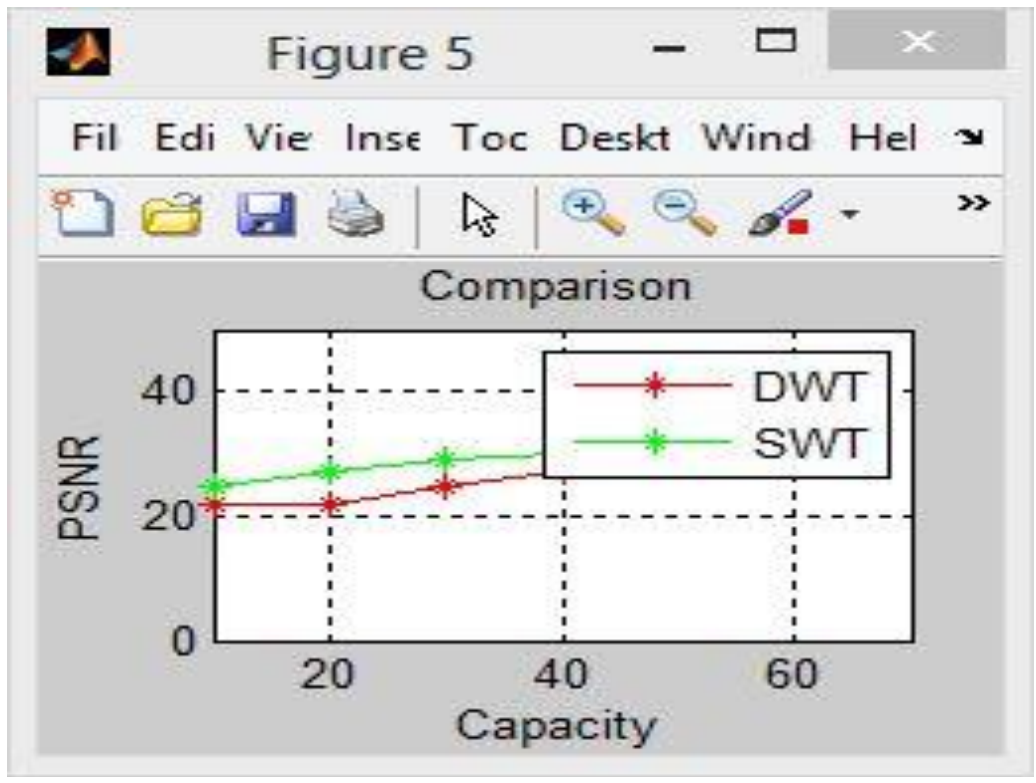

Figure 3.Comparison Graph of swt and dwt

\section{Conclusion}

In this paper, a video watermarking scheme which combines PCA with swt(bi-orthogonal wavelet) has been implemented. Applying PCA on SWT coefficients increases the robustness of the algorithm. So, the proposed scheme satisfies the requirement of imperceptibility and robustness for a feasible watermarking scheme. Furthermore, the advantage of the proposed scheme is that we can embed the watermark in LL band without the high degradation of the other DWT watermarking schemes. Experimental results show that the proposed scheme is robust against common video processing attacks. Since we are using SWT, the logo or watermark capacity can be increased to large extent.proposed scheme also show that it should have higher psnr value with lower MSE than other Wavelet Transforms

\section{References}

[1]. C. T. Hsu and J. L. Wu, "DCT-based watermarking for video," IEEETrans. Consumer Electron., vol. 44, no. 1, pp. 206-216, Feb. 1998.

[2]. L. Young-Yoon, J. Han-Seung, and L. Uk,“3D DFT-based videowatermarking using perceptual models,” in Proc. IEEE 46th Midwest Symp. Circuits Syst., vol. 3. Dec. 2003, pp. 1579-1583.

[3]. Xinghao Jiang, Tanfeng Sun, Jianhua Li and Ye Yun,"A Novel Real-Time MPEG-2 Video Watermarking Scheme in Copyright Protection", Digital Watermarking, Lecture Notes in Computer Science, Vol: 5450, pp:45-51, 2009.11

[4]. P. Campisi and A. Neri, "Perceptual video watermarking in the 3D-DWTdomain using a multiplicative approach," in Lecture Notes in ComputerScience, vol. 3710. New York, NY, USA: Springer-Verlag, Sep. 2005,pp. 432-443.

[5]. Xinxing Jing,Yan Liu, Xi Chen, Ping Zhou, The Domain Block Video watermarking Scheme Based video sequences' characteristics and DCT,2012.The 7th International Conference on Computer Science and Education. Australia5

[6]. Yan Liua and Jiying Zhao," A new video watermarking algorithm based on 1D DFT and Radon transform ",Signal Processing, Vol. 90, No. 2, pp. 626-639, 2010.13 